\title{
VLBA observations of Intraday Variable Sources
}

\section{Brian Moloney*University College, Cork}

E-mail: bmoloneyegmail.com

\section{Denise Gabuzda}

University College Cork

E-mail: gabuzda@physics.ucc.ie

\begin{abstract}
We present preliminary results of VLBA observations of a set of Intraday Variable sources. These sources were observed at 8 and 15-GHz by both the VLA and VLBA arrays in 1997. Our analysis yielded VLBA maps of these sources in both intensity and polarisation, while the VLA data provided information about the integrated variability of the sources during the VLBA run. These data indicate appreciable polarisation variability in several sources, which can be investigated by making VLBA Q and $\mathrm{U}$ maps for separate time intervals during the run. We present 15GHz polarisation maps of $0917+624$ which may suggest the region where the variability of this source originated. A comparison of the VLA and total VLBA polarisation shows that the VLA and VLBA variability of the sources during the VLBA run are correlated, demonstrating that the observed VLA variability is detected by the VLBA data
\end{abstract}

8th European VLBI Network Symposium

September 26-29, 2006

Toruń, Poland

${ }^{*}$ Speaker. 


\section{Introduction}

Intraday variability is the phenomenon in which the intensity and/or polarised flux of a source are observed to fluctuate on timescales of hours. There exist two main hypotheses to explain this behaviour.

The extrinsic variability hypothesis suggests that the observed variability arises from outside the source, namely in the interstellar medium. As the radio waves from an AGN travel through the interstellar medium, they are focused and defocused by inhomogeneities in the medium resulting in a pattern of bright and dark patches that are received at the Earth. This pattern is observed as rapid variability in the radio light curves.

This theory has gained credence from observations of several extreme IDV sources, in particular J1819+3845. J1819+3845 is one of the most extremely variable extragalactic sources known at radio wavelengths, exhibiting variations in excess of a factor of 10 at $5 \mathrm{GHz}(50-500 \mathrm{mJy})$ on a timescale of hours. Observations by Dennett-Thorpe \& De Bruyn [3] have shown an annual modulation in the characteristic timescale of this source which is clearly related to the Earth's orbit around the Sun. In addition, further observations established that the flux patterns observed at two widely separated antennas were identical but offset by a certain time-lag [4]. These two results leave no doubt that the variability observed in $\mathrm{J} 1819+3845$ is due to extrinsic effects.

The intrinsic variability hypothesis suggests that the variability observed arises within the source. However, this theory is restricted by relativistic constraints. If the variability arises from a single component its size can be deduced from the variation timescale and the speed of light. The resulting size constraints imply very high brightness temperatures, up to $10^{17} \mathrm{~K}$, which exceed the Inverse Compton limit of $\sim 10^{11}-10^{12} \mathrm{~K}$. This problem can be alleviated by invoking relativistic motion of the source roughly towards the Earth but this requires a Doppler factor of the order of 1000, which may pose theoretical difficulties. However, results obtained in a number of studies, e.g. evidence for correlated optical and radio variability in 0716+714 (Quirrenbach et al. 1991 [5]), or the detection of rapidly variable polarisation in individual compact VLBI components not accompanied by comparable variations in total intensity (Gabuzda et al. 2000 [1] [2]), suggest that it may be difficult to explain all observed IDV as being the result of extrinsic mechanisms.

\section{Observations}

Observations of six IDV sources $(0917+624,0954+658,1642+690,2007+777,0804+499$, $1150+812$ ) were made at 8 and 15-GHz by both the VLA and VLBA in June 1997. This contribution will only be concerned with the $15-\mathrm{GHz}$ data. It is also important to note that, while the VLA and VLBA observations were simultaneous, they were completely independent of one another.

The purpose of these observations was to further investigate the properties of these sources and their variability. Our aim was to establish which sources were varying, if any, and to try to establish if the variability could be pinpointed to a specific region within the observed structure. The first step was to analyse the VLA light-curves, and thus establish which sources were varying during the VLBA run. The preliminary calibration and reduction of the VLA and VLBA data, and the mapping of the VLBA data were done in AIPS using standard techniques. 

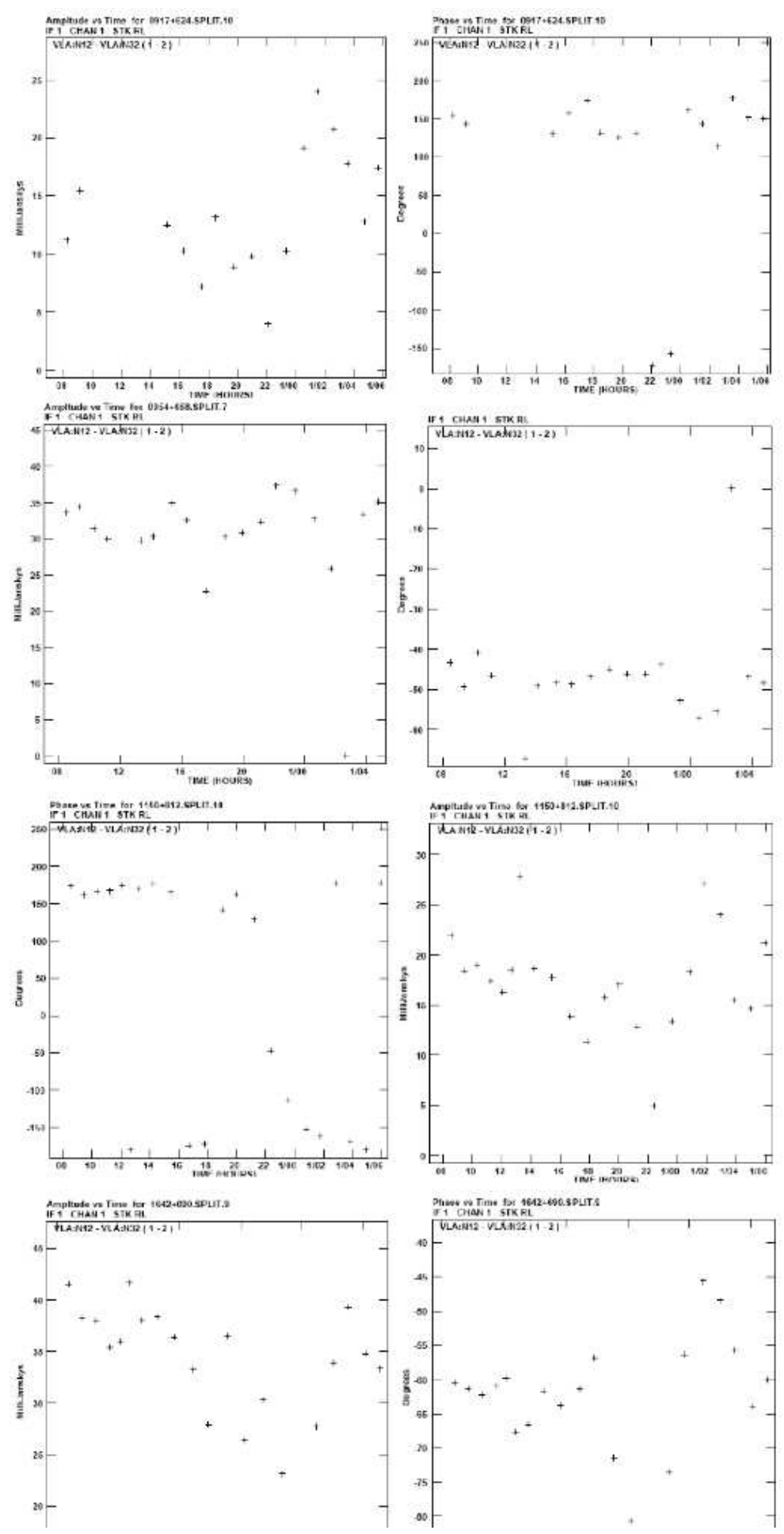

Figure 1: Polarisation amplitude (left) and phase (right) versus time for the VLA data for $0917+624$, $0954+658,1150+812$, and $1642+690$ (top to bottom). The AIPS task TBAVG was used to derive the amplitude and phase of the RL visibilities with an average time of 5 minutes. 


\section{Preliminary results}

The plots in Figure 1 show how the polarisation (RL) amplitude and phase angle of several sources evolved over the course of our observations. 0917+624 shows an amplitude change of a factor of two yet remains stable in polarisation angle. The source $0954+658$ remained quite stable in both amplitude and phase. Of the remaining sources, 1642+690 and 1150+812 exhibited the strongest variability; $1150+812$ is of particular interest as it displays what may have been a full rotation in the polarisation angle.

When investigating possible polarisation variations on VLBI scales, we make use of the fact that the intensity variations are much less than those observed in polarised flux. Because of this, we are able to make the intensity maps with some degree of confidence that the variability does not affect them in a significant way. Then, using the final intensity map, we can subdivide the data into a number of separate time intervals, from which we can obtain polarisation maps that correspond to certain periods of time. These intervals were chosen by looking at the VLA plots from Figure 1. Of course, the minimum time interval that can be studied is constrained by the corresponding uv-coverage, and the need to have sufficient data to obtain a reasonable polarisation map. Note however, that the self-calibration of all four correlations (RR, LL, RL, LR) is based on gain solutions obtained using all the total intensity data, so that the uv-coverage used to obtain these gain solutions is very good.

\section{$3.10917+624$}

The total intensity map of 0917+624 obtained using all our VLBA data is shown in Figure 2. The intensity map looks as expected with a jet emerging to the North. Though this map was made for the entire dataset, and is subject to the corruption of the polarisation data due to variability during the run, the predominant polarisation structure is clearly visible. The dominant magnetic field is transverse in both the core and outer jet, suggesting the jet may have a helical $\vec{B}$-field with a dominant toroidal component.

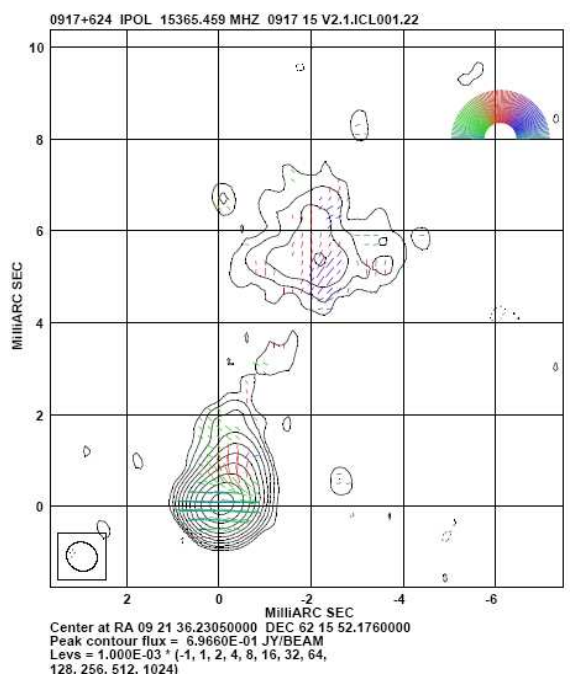

Figure 2: Intensity map (using all the data) 
This source underwent a jump in polarised flux from $\sim 10$ to $\sim 20$ mJy while remaining constant in polarisation angle. These two states defined the intervals that we chose $(16 \rightarrow 1 / 0,1 / 0 \rightarrow$ 1/6), and the corresponding maps are shown in Figure 3.
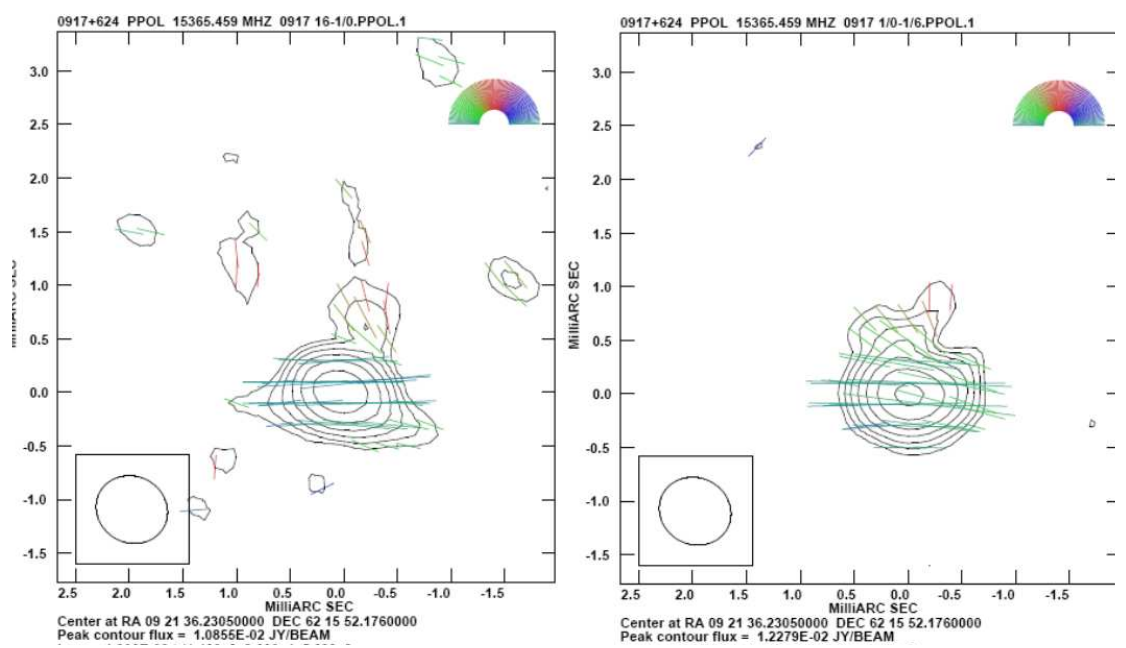

Figure 3: Time-defined polarisation maps

The $1^{\text {st }}$ polarisation image shows two dominant components situated at the core and $\sim(-0.3,0.7)$ mas. However, the second map shows a clear suggestion of a $3^{r d}$ component located at $\sim(0.3,0.6)$; perhaps this component is the source of the VLA polarised flux variation. If this is true, we must do further analysis to determine what this means with regard to the source of the variability (intrinsic/ extrinsic) and its properties.

\subsection{Vector subtraction}

To test whether any variability observed on VLBA scales was physically significant we performed a vector subtraction of the polarisation vectors for the VLA and VLBA scales i.e. $\vec{P}_{\text {diff }}=$ $\vec{P}_{V L A}-\vec{P}_{V L B A}$. If there are variations on the VLBA scale then this value should yield a constant vector. The calculation of the polarisation vector was done as follows:

- VLA: Using the task LISTR, we measured the average flux and angle for the time intervals that we chose.

- VLBA: Using the stokes ' $Q$ ' and ' $U$ ' maps in combination with the tasks CCMRG \& PRTCC we calculated the sum of the clean components of each map. The polarisation amplitude and orientation are then given by $P=\sqrt{Q^{2}+U^{2}}$ and $2 \chi=\tan ^{-1} \frac{U}{Q}$, respectively.

The following figure 4 shows these results for $0917+624$ and $2007+777$ as examples. It is quite clear that the difference between the two vectors, $\vec{P}_{\text {diff }}$, does appear to be constant for the time intervals chosen which can be more clearly seen in Figure 5. This demonstrates that the integrated variations were detected in the VLBA data, providing hope that our further analysis of these data will enable us to identify individual varying regions in the VLBA structure. 


\begin{tabular}{|c||c|c|c|c||c|c|}
\hline$\underline{0917+624}$ & $\vec{P}_{V L B A}$ & $\chi_{V L B A}$ & $\vec{P}_{V L A}$ & $\chi_{V L A}$ & $\vec{P}_{\text {diff }}$ & $\chi_{\text {diff }}$ \\
$16 \rightarrow 1 / 0$ & 8 & -88 & 13 & 77 & 7 & -30 \\
$1 / 0 \rightarrow 1 / 6$ & 14 & 85 & 22 & 76 & 10 & -27 \\
& & & & & & \\
$\frac{2007+777}{8 \rightarrow 16}$ & & & & & & \\
$16 \rightarrow 21$ & 60 & -67 & 92 & -70 & 14 & 7 \\
$21 \rightarrow 1 / 7$ & 80 & -65 & 78 & -65 & 16 & 23 \\
\hline
\end{tabular}

Figure 4: Calculation of the intermediate-scale polarisation vector

Polarisation Amplitude vs Time for $2007+777$

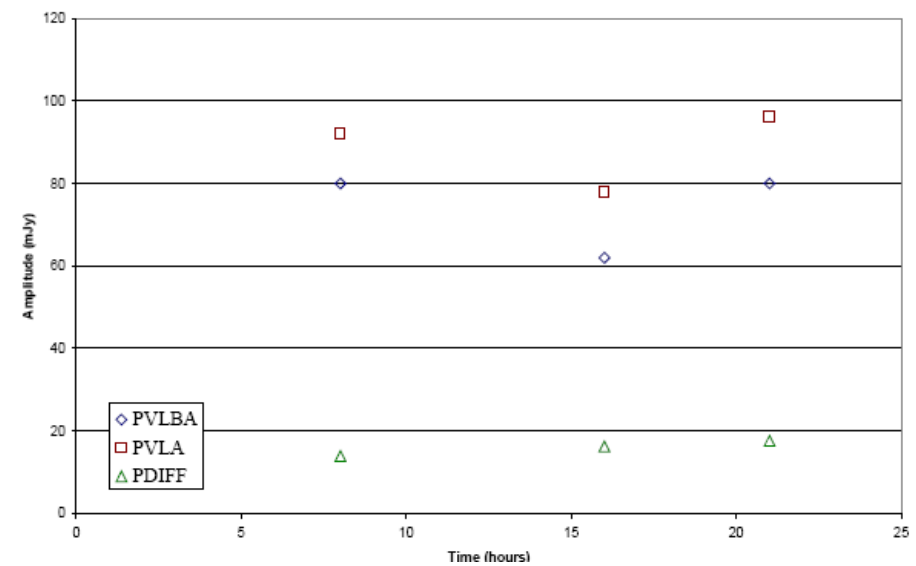

Figure 5: Polarisation amplitude vs time for $2007+777$

\subsection{Future work}

Our analysis of these data is ongoing. An important part of this analysis will be model-fitting of the VLBA total intensity and polarisation data for individual time intervals. This will enable us to ascertain more reliably whether some specific region(s) in the VLBA structure were varying during our VLBA run. The observed properties of these regions can then be compared with the expectations of scintillation models for the observed variability, to determine whether or not intrinsic variations are required to explain the observed polarisation variability.

\section{References}

[1] Gabuzda, D. C., Kochenov, P. YU., Cawthorne, T. V. 2000 MNRAS 319, 1125

[2] Gabuzda, D. C., Kochenov, P. YU., Kollgaard, R. I., Cawthorne, T. V. 2000, MNRAS 315, 229

[3] Dennett-Thorpe J., De Bruyn A.G. 2003, A\&A 404, 113

[4] Dennett-Thorpe J., De Bruyn A.G. 2002, Nature 415, 57

[5] Quirrenbach, A., Witzel, A., Wagner, S., Sanchez-Pons, F., Krichbaum, T. P., Wegner, R., Anton, K., Erkens, U., Haehnelt, M., Zensus, J. A., Johnston, K. J. 1991, ApJ 372, L71 